\title{
Planificación y gestión del tiempo académico de estudiantes universitarios
}

\author{
Nicolás Reyes-González ${ }^{{ }^{*}}$, Alba L. Meneses-Báez ${ }^{1}$ y Alejandro Díaz-Mujica ${ }^{2}$ \\ (1) Universidad Católica de Colombia, Facultad de Psicología, Bogotá-Colombia. \\ (correo-e: nicolasreye@gmail.com; almeneses@ucatolica.edu.co) \\ (2) Universidad de Concepción, Departamento de Psicología, Concepción-Chile (correo-e: adiazm@udec.cl) \\ * Autor a quien debe ser dirigida la correspondencia.
}

Recibido Jun. 16, 2021; Aceptado Ago. 12, 2021; Versión final Sep. 17, 2021, Publicado Feb. 2022

\begin{abstract}
Resumen
Esta investigación evalúa y caracteriza las estrategias para planificación y uso del tiempo de universitarios. Se implementa un estudio de caso mixto y una encuesta abierta a 72 estudiantes universitarios de primer y segundo año. Se aplica la sub-escala de planificación del inventario de habilidades meta-cognitivas (MAI) adaptado. Los resultados muestran que no se presentan diferencias significativas entre sexo, jornada, compromiso horario laboral, previos estudios universitarios, formulación de objetivos y desempeño académico. Los análisis textuales para estudiantes de alto y bajo rendimiento académico presentan diferencias en las estrategias de planificación y priorización del tiempo, herramientas de apoyo y formulación de objetivos de estudio. Se concluye que hay diferencias en la descripción de las estrategias y herramientas de los estudiantes para la planificación del tiempo de estudio de acuerdo al rendimiento académico y las variables sexo, previos estudios universitarios, formulación de objetivos de estudio, jornada y compromiso horario laboral.
\end{abstract}

Palabras clave: gestión; tiempo; educación superior; planificación; universidad

\section{Planning and academic time management of university students}

\begin{abstract}
This study evaluates and characterizes planning and time management strategies of university students. The methodology is mixed, quantitative and qualitative. Seventy-two first and second year university students are surveyed by applying an adapted planning sub-scale: metacognitive awareness inventory (MAI). The results show that there are no significant differences between gender, working hours, commitment to work, previous university studies, formulation of objectives, and academic performance. Text analysis for students with high and low academic performances show differences in time planning and prioritization strategies, support tools, and formulation of study objectives. In conclusion, there are differences in student descriptions of planning and time management tools and strategies depending on their academic performances and on the following variables: gender, working hours, commitment to work, previous university studies, and formulation of objectives.
\end{abstract}

Keywords: time management; higher education; planning; university 


\section{INTRODUCCIÓN}

El éxito académico en estudiantes universitarios ha estado asociado a indicadores de permanencia, rendimiento y ajuste (Robbins et al., 2004; Credé y Niehorster 2012; Fong et al., 2017). Dentro de los elementos asociados al rendimiento, se encuentran las estrategias para la regulación del aprendizaje (DíazMujica et al., 2019; Pérez et al., 2010) que refieren al uso auto-direccionado de pensamientos, sentimientos y acciones para incrementar la motivación al aprendizaje (Britton y Tesser, 1991; Zimmerman, 2002; De la Fuente et al., 2017) y que refieren a componentes propios de la disposición al aprendizaje, cognitivos y metacognitivos (Zimmerman y Bandura, 1994; Pérez et al., 2013). La gestión del tiempo académico es un elemento que permite la disposición al aprendizaje y se asocia a elementos de la regulación de la cognición. Se orienta a las conductas que permiten el uso efectivo del tiempo para la obtención de actividades dirigidas a una meta y se asocia con el establecimiento de objetivos y asignación de tiempos, recursos y estrategias para su cumplimiento (Schraw y Denninson, 1994; Pérez et al., 2013; Garzón Umerenkova y Flores, 2018)

Al respecto, se ha identificado el efecto que tiene la gestión del tiempo académico con los resultados de desempeño y logro académico (Credé y Kuncel, 2008; Umerenkova y Flores, 2018). Teniendo en cuenta lo anterior, previas investigaciones han resaltado la importancia que tienen las estrategias y herramientas en las que se apoyan los universitarios para la planificación del tiempo académico a la luz de estrategias de intervención orientadas a desarrollo de habilidades (Robbins et al., 2004; Pérez et al., 2010). Adicionalmente, se han identificado elementos que influyen en la efectividad de las estrategias de planificación y uso de tiempo en estudiantes universitarios (López-Angulo et al., 2020). En primer lugar, previos estudios han identificado que las mujeres suelen establecer con mayor frecuencia metas y actividades de organización; mayor gestión del tiempo y organización del estudio; y mayor programación respecto a las demandas académicas (Soares, et al., 2011; Durán-Aponte y Pujol, 2013; Elvira-Valdés y Pujol, 2012). Así mismo, que los hombres tienden presentar mayores conductas de procrastinación (Garzón-Umerenkova y Gil-Flores, 2017).

Por otro lado, existe influencia del tiempo destinado para el estudio en la adaptación a las demandas del proceso educativo, es así que los estudiantes de tiempo parcial disponen de menos horas para el estudio y requieren de mayor habilidad para la planificación (Carney et al., 2005). El estudio de Robotham (2012), muestra que los estudiantes que disponen de menor dedicación horaria al estudio requieren mayores habilidades para la administración efectiva. Así mismo Garzón-Umerenkova y Gil-Flores (2017) sugieren que, el incremento de responsabilidades adicionales a las académicas tiene un efecto en las conductas de procrastinación debido a que, la demanda de actividades es un obstáculo para el cumplimiento de los deberes de estudio.

Así mismo, la existencia de actividades laborales paralelas a las académicas, influye en la efectividad de la planificación del tiempo académico (Sainz et al., 2019). Robotham (2012) identificó que una ocupación mayor a 20 horas por semana a actividad laboral influye en el desempeño académico. Garzón-Umerenkova y GilFlores (2017) mostraron que los estudiantes que combinan la actividad laboral y la actividad académica incrementan la procrastinación e identifican la actividad laboral como una dificultad en el cumplimiento de las metas académicas. Los estudiantes que laboran disponen de menor tiempo y por tanto requieren de mayores habilidades para la planificación y el uso efectivo del tiempo académico. Finalmente, el estudio de Crede y Niehorster (2012), ha identificado que las experiencias previas a nivel universitario tienen una influencia respecto a las estrategias para el afrontamiento de las demandas académicas. Dichas experiencias, tanto favorables como desfavorables, determinan las estrategias implementadas para cumplir con los deberes académicos. Por esta razón las estrategias de planificación y uso del tiempo de los estudiantes que han realizado estudios previos pueden diferir respecto a los que toman por primera vez sus estudios universitarios.

Teniendo en cuenta lo anterior, el presente estudio tiene por objetivo evaluar las habilidades de planificación de estudiantes universitarios y describir cuáles son las estrategias de gestión del tiempo que implementan los alumnos con menor y mayor habilidad de planificación de acuerdo con el rendimiento académico. Así mismo y teniendo en cuenta las variables que pueden afectar esas estrategias, se busca contrastar las acciones de planificación de acuerdo con el sexo, la condición laboral, la jornada de estudio, la formulación de objetivos de estudio y la existencia de estudios universitarios previos.

\section{MÉTODO}

Se implementó un estudio de caso de método mixto (Creswell y Plano Clark, 2018) de metodología cuantitativa a cualitativa para identificar cuáles son las prácticas de los estudiantes de primer y segundo año, de acuerdo con las variables objetivo.

\section{Población}

Se aplicó a una muestra por conveniencia de 72 estudiantes de tiempo parcial en primer y segundo año de pregrado de la facultad de psicología de la Universidad Católica de Colombia. 78\% corresponde a mujeres con edad promedio de 20.5 años ( $D S=3.5$ ) y $22 \%$ hombres con 21.5 años $(D S=3.9)$. $60 \%$ corresponde a 
alumnos que toman clase en la jornada del día (entre 7 y 16 horas) y de la muestra $34.7 \%$ labora. Los estudiantes estaban matriculados, su participación fue voluntaria, tenían asignación académica completa y por su participación no obtuvieron ningún incentivo.

\section{Instrumentos}

Se aplicó la sub-escala de planificación del Inventario de Habilidades Metacognitivas (MAI) (Schraw y Denninson, 1994) la cual fue adaptada para población colombiana (Huertas et al., 2014). Tiene un índice de consistencia interna alfa de Cronbach de 0.76 (Huertas et al., 2014) y para el presente estudio de 0.71 , lo cual se considera aceptable (Cortina, 1993). Consta de siete reactivos de opción múltiple, única respuesta y escala Likert de 5 niveles que van de completamente en desacuerdo a completamente de acuerdo. Algunos ejemplos de preguntas son "mientras estudio organizo el tiempo para poder acabar la tarea" o "me propongo objetivos específicos antes de empezar una tarea"

Encuesta de pregunta abierta de actividades y planificación del tiempo que evaluó las estrategias y prácticas para planeación y uso del tiempo académico. Se elaboró a partir de las líneas temáticas utilizadas por Umerenkova y Flores (2018) con base en cinco preguntas sobre las estrategias para planificación del tiempo, herramientas de apoyo, formulación de objetivos de estudio y estrategias de priorización. Adicionalmente se solicitó a los estudiantes un auto-reporte sobre promedio académico indicando el promedio acumulado actual y del periodo anterior.

\section{Procedimiento}

El procedimiento se realizó en cuatro fases. Durante la primera se procedió a la gestión y autorización de las directivas institucionales para la participación de la muestra de estudiantes. En la segunda fase se seleccionó la escala y se diseñaron las preguntas de la encuesta de pregunta abierta, las cuales fueron sometidas a revisión de contenido por expertos y a entrevistas cognitivas con el fin corregir posibles dificultades de las preguntas (Caicedo y Zalazar-Jaime, 2018). Este proceso se realizó en dos pasos 1) diligenciamiento del cuestionario por 4 estudiantes universitarios y 2) entrevista semiestructurada a 2 de ellos. En la fase tres y con previa autorización por parte de los estudiantes a través de consentimiento informado, se realizó la aplicación de los instrumentos a través de formularios virtuales. Finalmente, en la fase cuatro se realizó el proceso de análisis de datos.

\section{Análisis de información}

Los análisis comparativos se aplican con el fin de contrastar los resultados para la escala de planificación y las respuestas dadas a la encuesta semiestructurada para las variables sexo, jornada, situación laboral, experiencia universitaria previa, formulación de objetivos y desempeño académicos. Teniendo en cuenta lo anterior, se aplicaron tres análisis.

Primero, una prueba $U$ Mann-Whitney para muestras independientes que se aplicó teniendo en cuenta que los resultados en la escala del MAI no siguen una distribución normal según prueba de Kolmogorov-Smirnov $(p=0.007$, media $=3.9, D S=0.50)$, este realizó con el software SPSS V.27. Para los análisis respectivos al desempeño académico, se construyeron dos sub-muestras, la primera (grupo bajo) con el grupo de estudiantes que reportaron promedios en el cuartil uno y la segunda (grupo alto) con los que reportaron promedios reportados en los cuartiles tres y cuatro. Segundo, un análisis textual con el método de especificidades, este para observar las diferencias en las respuestas reportadas por los participantes a las preguntas abiertas. Se realizó con el Software Spad 9.1 y, partir de la construcción de un sub-corpus de texto para cada grupo, contrastó la existencia de vocablos característicos de los grupos a comparar (Flores et al., 1996). Finalmente, y para describir las estrategias de uso y planificación del tiempo de los participantes de alto y bajo rendimiento de acuerdo con los puntajes obtenidos en la sub-escala de planificación del MAI, se aplicó un análisis textual de correspondencias múltiples (ACM en adelante) también con el Software Spad 9.1.

\section{RESULTADOS}

Los resultados se presentarán para la muestra del estudio y teniendo en cuenta los análisis de diferencia de medias los cuales fueron complementados con los resultados obtenidos en el método de las especificidades cuya frecuencia de palabras en el sub-corpus de cada grupo objeto de comparación se presenta en las tablas incluidas en los anexos.

\section{Sexo}

Los resultados presentados en la tabla 1 muestra que no se observaron diferencias significativas de acuerdo con el sexo en la escala de planificación $(z=-0.647 ; p=0.51)$. Los datos presentados en la tabla 2 muestran las diferencias a partir del método de especificidades. Se observó en los hombres estrategias relacionadas con madrugar para estudiar fuera de clase; hacer uso de herramientas como cuadernos, lecturas, reloj, 
calendarios o apuntes y hacer uso de estrategias de priorización de acuerdo con las características de los trabajos. Por otro lado, las mujeres reportaron estrategias de planificación asociadas la organización y de priorización asociadas a la organización, establecimiento de fechas y disposición de tiempo y los tipos de tareas o actividades para priorizar. No se identificaron diferencias en los sub-corpus respecto a los aspectos que se tienen en cuenta en la formulación de objetivos de estudio.

Tabla 1: Estadísticos descriptivos y resultados prueba diferencia de medias

\begin{tabular}{|c|c|c|c|c|c|c|}
\hline & & \multicolumn{3}{|c|}{ Descriptivos } & \multicolumn{2}{|c|}{ Diferencia de Medias } \\
\hline & & $N$ & Media & $D S$ & U Mann-Whitney (z) & Sig \\
\hline \multirow{2}{*}{ Sexo } & Hombre & 16 & 3.80 & 0.50 & \multirow[t]{2}{*}{-0.647} & \multirow[t]{2}{*}{0.518} \\
\hline & Mujer & 56 & 3.94 & 0.51 & & \\
\hline \multirow{2}{*}{ Jornada } & Diurna & 43 & 3.93 & 0.54 & \multirow[t]{2}{*}{-0.433} & \multirow[t]{2}{*}{0.665} \\
\hline & Nocturna & 29 & 3.93 & 0.45 & & \\
\hline \multirow{2}{*}{$\begin{array}{l}\text { Compromiso horario de actividad } \\
\text { laboral }\end{array}$} & Trabaja & 25 & 3.96 & 0.48 & \multirow[t]{2}{*}{-0.042} & \multirow[t]{2}{*}{0.967} \\
\hline & No trabaja & 47 & 3.90 & 0.52 & & \\
\hline \multirow{2}{*}{ Antecedentes de estudios universitarios } & Sí & 22 & 3.81 & 0.63 & \multirow[t]{2}{*}{-0.977} & \multirow[t]{2}{*}{0.329} \\
\hline & No & 50 & 3.98 & 0.44 & & \\
\hline \multirow{2}{*}{ Promedio Acumulado } & Alto & 26 & 3.83 & 0.52 & \multirow[t]{2}{*}{-0.946} & \multirow[t]{2}{*}{0.344} \\
\hline & Bajo & 26 & 3.94 & 0.62 & & \\
\hline \multirow{2}{*}{ Promedio Semestre Anterior } & Alto & 23 & 3.83 & 0.55 & \multirow[t]{2}{*}{-0.757} & \multirow[t]{2}{*}{0.449} \\
\hline & Bajo & 30 & 3.93 & 0.61 & & \\
\hline \multirow{2}{*}{ Formulación de objetivos de estudio } & Sí & 42 & 3.96 & 0.53 & \multirow[t]{2}{*}{-0.740} & \multirow[t]{2}{*}{0.459} \\
\hline & No & 30 & 3.88 & 0.49 & & \\
\hline
\end{tabular}

Tabla 2: Método especificidades comparación por sexo

\begin{tabular}{|c|c|c|c|c|c|c|c|}
\hline \multirow{2}{*}{ Dimensión } & \multicolumn{3}{|c|}{ Hombre } & \multirow{2}{*}{ Dimensión } & \multicolumn{3}{|c|}{ Mujer } \\
\hline & Palabra & F. Interna & F. Global & & Palabra & F. Interna & F. Global \\
\hline $\begin{array}{l}\text { Estrategias de } \\
\text { planificación }\end{array}$ & Madrugar & 3 & 4 & $\begin{array}{l}\text { Estrategias de } \\
\text { planificación }\end{array}$ & Organizar & 20 & 21 \\
\hline \multirow{5}{*}{$\begin{array}{l}\text { Herramientas de apoyo } \\
\text { para administrar el } \\
\text { tiempo }\end{array}$} & Leer & 2 & 2 & \multirow{6}{*}{$\begin{array}{l}\text { Estrategias de } \\
\text { priorización }\end{array}$} & Organizo & 17 & 17 \\
\hline & Cuaderno & 2 & 2 & & Tiempo & 30 & 33 \\
\hline & Reloj & 2 & 2 & & Actividades & 25 & 28 \\
\hline & Apuntes & 3 & 6 & & Tareas & 8 & 8 \\
\hline & Calendario & 3 & 11 & & & & \\
\hline $\begin{array}{l}\text { Estrategias de } \\
\text { priorización }\end{array}$ & Trabajos & 5 & 12 & & Fecha & 7 & 7 \\
\hline
\end{tabular}

\section{Jornada}

No se observaron diferencias significativas en la escala de planificación ( $z=-0.433, p=0.665)$ (ver tabla 1). Los resultados obtenidos a partir del método de las especificidades presentados en la tabla 3 , muestran en los estudiantes de la jornada diurna menor diversidad de estrategias para planificación. Estas relativas al establecimiento de prioridades; criterios de organización de acuerdo con la dificultad, alcances o límites de las actividades e investigar respecto a trabajos y tareas a realizar. Se observó uso de herramientas de apoyo como libros, alarma, cronómetros, notas o post, guías y herramientas de internet. Los objetivos de estudio se asocian a la dificultad de las tareas a la intención de mejorar, al establecimiento de alcances, y se formulan de acuerdo con la disposición de tiempo y lograr el cumplimiento de las actividades. Respecto a las estrategias para la priorización consideran relevante dejar tiempo para descansar.

Los estudiantes de jornada nocturna reportaron estrategias de planificación orientadas a la organización, las características de las actividades o temas; usan notas; y consideran horarios para estudiar en la madrugada, mañana o noche; reportaron tener en cuenta la frecuencia de las actividades estudio ya sea diarias 0 semanales y reportaron uso de tutorías y agenda de actividades. Se observó uso de herramientas como agendas o ninguna herramienta para apoyar la planificación. En los objetivos de estudio, se identificó uso de criterios orientados por las características de las actividades y la distribución de labores, temas o trabajos. Como estrategias de priorización se observó el uso de criterios acordes con el tipo de actividad, suelen organizar las actividades por prioridad, con base en entregas, tiempos disponibles y a través del uso de agendas. También consideran como estrategia para priorizar facilitar la concentración en las actividades académicas. 
Tabla 3: Método especificidades, jornada

\begin{tabular}{|c|c|c|c|c|c|c|c|}
\hline \multicolumn{4}{|c|}{ Nocturna } & \multicolumn{4}{|c|}{ Diurna } \\
\hline Dimensión & Palabra & F. Interna & F. Global & Dimensión & Palabra & F. Interna & F. Global \\
\hline \multirow{11}{*}{$\begin{array}{l}\text { Estrategias de } \\
\text { planificación }\end{array}$} & Organizar & 14 & 21 & \multirow{4}{*}{$\begin{array}{l}\text { Estrategias de } \\
\text { planificación }\end{array}$} & Prioridad & 8 & 10 \\
\hline & Notas & 3 & 3 & & Dificultad & 3 & 3 \\
\hline & Mañana & 3 & 3 & & Limite & 3 & 3 \\
\hline & Noche & 3 & 3 & & Investigar & 3 & 3 \\
\hline & Actividades & 12 & 21 & \multirow{7}{*}{$\begin{array}{l}\text { Herramientas de } \\
\text { apoyo para } \\
\text { administrar el } \\
\text { tiempo }\end{array}$} & Libros & 6 & 7 \\
\hline & Diario & 6 & 9 & & Alarmas & 3 & 3 \\
\hline & Tutorías & 2 & 2 & & Cronometro & 3 & 3 \\
\hline & Temas & 2 & 2 & & Ayudas & 3 & 3 \\
\hline & Agenda & 3 & 4 & & Post & 3 & 3 \\
\hline & Madrugar & 3 & 4 & & Guías & 3 & 3 \\
\hline & Semana & 5 & 9 & & Internet & 2 & 3 \\
\hline \multirow{2}{*}{$\begin{array}{l}\text { Herramientas de apoyo } \\
\text { para administrar el } \\
\text { tiempo }\end{array}$} & Agenda & 6 & 7 & \multirow{6}{*}{$\begin{array}{l}\text { Elementos que } \\
\text { tiene en cuenta al } \\
\text { formular objetivos }\end{array}$} & Dificultad & 2 & 2 \\
\hline & Ninguna & 4 & 4 & & Mejorar & 2 & 2 \\
\hline \multirow{4}{*}{$\begin{array}{l}\text { Elementos que tiene en } \\
\text { cuenta al formular } \\
\text { objetivos }\end{array}$} & Actividades & 6 & 6 & & Alcance & 3 & 4 \\
\hline & Distribuir & 4 & 4 & & Tiempo & 8 & 14 \\
\hline & Tema & 6 & 8 & & \multirow{2}{*}{ Cumplir } & \multirow{2}{*}{4} & \multirow{2}{*}{6} \\
\hline & Trabajo & 7 & 10 & & & & \\
\hline \multirow{9}{*}{$\begin{array}{l}\text { Estrategias de } \\
\text { priorización }\end{array}$} & Prioridad & 5 & 6 & \multirow{9}{*}{$\begin{array}{l}\text { Estrategias de } \\
\text { priorización }\end{array}$} & \multirow{9}{*}{ Descanso } & \multirow{9}{*}{4} & \multirow{9}{*}{4} \\
\hline & Entrega & 12 & 20 & & & & \\
\hline & Tareas & 6 & 8 & & & & \\
\hline & Buscar & 3 & 3 & & & & \\
\hline & Concentración & 3 & 3 & & & & \\
\hline & Agenda & 2 & 2 & & & & \\
\hline & Tranquilidad & 2 & 2 & & & & \\
\hline & Organizo & 9 & 17 & & & & \\
\hline & Tiempo & 15 & 33 & & & & \\
\hline
\end{tabular}

\section{Compromiso horario de actividad laboral}

Tener compromiso horario de actividad laboral no mostró diferencias significativas en la prueba de planificación MAI $(\mathrm{z}=-0.042, \mathrm{p}=0.967)$ (ver tabla 1). El análisis textual mostró menor diversidad de estrategias para la planificación del tiempo en los estudiantes que no tienen esta condición. Reportan estrategias que se rigen por el uso del tiempo y las labores pendientes. Se apoyan en herramientas como el computador, el celular, las listas de tareas (vocablo "chulear"), el apoyo de compañeros y el uso del correo electrónico. Para formular objetivos de estudio tienen en cuenta la distribución del tiempo, resolver las labores pendientes acorde con lo que pueden realizar y genera mayor interés. La priorización se da con base en la importancia de la actividad o tarea y lo que pueden realizar. También consideran importante que la priorización permita el descanso (ver tabla 4).

Los estudiantes que tienen compromiso de horario laboral reportaron mayor cantidad de estrategias para administrar el tiempo, se observa el uso de acciones como la organización y planificación de actividades y tareas. También se observa que contemplan organización de actividades en horarios de la mañana o en la noche, y forma periódica acudiendo a actividades semanales o diarias. Estas estrategias se formulan teniendo en cuenta la importancia o haciendo uso de notas o agenda de actividades. Las herramientas de apoyo para la planificación implementadas por este grupo, corresponde a agendas, notas, calendarios, internet y planeadores; algunos no usan apoyos. Los elementos de objetivos de estudio en este grupo se orientan a la distribución del tiempo, la planificación de actividades, la profundización e indagación de temas y están orientados a las entregas y logros. La priorización la realizan con base en las características de las tareas, la carga y se busca la distribución de actividades, por medio de planeadores o agendas que permitan la organización y la concentración. 
Tabla 4: Método especificidades, compromiso horario de actividad laboral

\begin{tabular}{|c|c|c|c|c|c|c|c|}
\hline \multicolumn{4}{|c|}{ Tiene compromiso horario de actividad laboral } & \multicolumn{4}{|c|}{ No Tiene compromiso horario de actividad laboral } \\
\hline Dimensión & Palabra & F. Interna & F. Global & Dimensión & Palabra & F. Interna & F. Global \\
\hline \multirow{10}{*}{$\begin{array}{l}\text { Estrategias de } \\
\text { planificación }\end{array}$} & Actividades & 14 & 21 & \multirow{10}{*}{$\begin{array}{l}\text { Estrategias de } \\
\text { planificación }\end{array}$} & \multirow{3}{*}{ Pendiente } & \multirow{3}{*}{3} & \multirow{3}{*}{3} \\
\hline & Agenda & 4 & 4 & & & & \\
\hline & Organizar & 12 & 21 & & & & \\
\hline & Mañana & 3 & 3 & & \multirow{4}{*}{ Investigar } & \multirow{4}{*}{3} & \multirow{4}{*}{3} \\
\hline & Noche & 3 & 3 & & & & \\
\hline & Notas & 3 & 3 & & & & \\
\hline & Semana & 6 & 9 & & & & \\
\hline & Diario & 6 & 9 & & \multirow{3}{*}{ Tiempo } & \multirow{3}{*}{21} & \multirow{3}{*}{30} \\
\hline & Tareas & 8 & 16 & & & & \\
\hline & Importantes & 3 & 5 & & & & \\
\hline \multirow{6}{*}{$\begin{array}{l}\text { Herramientas de } \\
\text { apoyo para } \\
\text { administrar el } \\
\text { tiempo }\end{array}$} & Agenda & 5 & 7 & \multirow{6}{*}{$\begin{array}{l}\text { Herramientas } \\
\text { de apoyo para } \\
\text { administrar el } \\
\text { tiempo }\end{array}$} & Computador & 8 & 9 \\
\hline & Ninguna & 3 & 4 & & Celular & 11 & 13 \\
\hline & Notas & 2 & 2 & & Chuleando & 1 & 1 \\
\hline & Calendario & 5 & 11 & & Compañeros & 1 & 1 \\
\hline & Internet & 2 & 3 & & Correo & \multirow{2}{*}{1} & \multirow{2}{*}{1} \\
\hline & Planeador & 3 & 6 & & Correo & & \\
\hline \multirow{8}{*}{$\begin{array}{l}\text { Elementos que } \\
\text { tiene en cuenta al } \\
\text { formular objetivos }\end{array}$} & Distribuir & 4 & 4 & \multirow{8}{*}{$\begin{array}{l}\text { Elementos que } \\
\text { tiene en cuenta } \\
\text { al formular } \\
\text { objetivos }\end{array}$} & Pendientes & 4 & 4 \\
\hline & Profundizar & 2 & 2 & & Tiempo & 10 & 14 \\
\hline & Indagar & 2 & 2 & & Poder & 2 & 3 \\
\hline & Entrega & 2 & 2 & & \multirow{5}{*}{ Interés } & \multirow{5}{*}{2} & \multirow{5}{*}{3} \\
\hline & Lograr & 2 & 2 & & & & \\
\hline & Tema & 5 & 8 & & & & \\
\hline & Planificar & 3 & 4 & & & & \\
\hline & Tareas & 2 & 3 & & & & \\
\hline \multirow{7}{*}{$\begin{array}{l}\text { Estrategias de } \\
\text { priorización }\end{array}$} & Tareas & 6 & 8 & \multirow{7}{*}{$\begin{array}{l}\text { Estrategias de } \\
\text { priorización }\end{array}$} & Importancia & 7 & 8 \\
\hline & Concentración & 3 & 3 & & Descanso & 4 & 4 \\
\hline & Carga & 3 & 3 & & \multirow{5}{*}{ Puedo } & \multirow{5}{*}{4} & \multirow{5}{*}{4} \\
\hline & Distribuimos & 2 & 2 & & & & \\
\hline & Planeador & 2 & 2 & & & & \\
\hline & Agenda & 2 & 2 & & & & \\
\hline & Organizo & 8 & 17 & & & & \\
\hline
\end{tabular}

\section{Antecedentes de estudios universitarios}

Tener previos estudios universitarios previos no tuvo diferencias significativas en los puntajes de la escala de planificación ( $z=-0.977, p=0.329$ ) (ver tabla 1). Sin embargo, el análisis textual mostró que, para la muestra tomada, no tener antecedentes universitarios tiene menor cantidad de estrategias de planificación (ver tabla 5). Las estrategias se enfocan en los pendientes y lo que deben realizar con mayor urgencia. Las herramientas de apoyo son el computador, calendarios y notas. Al formular objetivos tienen en cuenta la carga, el tipo de actividades y el tiempo disponible. Priorizan con base en las características de las actividades, la complejidad, la urgencia y los trabajos por realizar. Esto, con el objetivo de organizar el trabajo a través de horarios.

Tabla 5: Método especificidades, antecedentes de estudios universitarios

\begin{tabular}{|c|c|c|c|c|c|c|c|}
\hline \multicolumn{4}{|c|}{ Tiene antecedentes de estudios universitarios } & \multicolumn{4}{|c|}{ No tiene antecedentes de estudios universitarios } \\
\hline Dimensión & Palabra & F. Interna & F. Global & Dimensión & Palabra & F. Interna & F. Global \\
\hline \multirow{16}{*}{$\begin{array}{l}\text { Estrategias de } \\
\text { planificación }\end{array}$} & Noche & 3 & 3 & \multirow{16}{*}{$\begin{array}{l}\text { Estrategias de } \\
\text { planificación }\end{array}$} & \multirow{8}{*}{ Trabajos } & \multirow{8}{*}{12} & \multirow{8}{*}{14} \\
\hline & Notas & 3 & 3 & & & & \\
\hline & Mañana & 3 & 3 & & & & \\
\hline & Tareas & 8 & 16 & & & & \\
\hline & Agenda & 3 & 4 & & & & \\
\hline & Dividir & 2 & 2 & & & & \\
\hline & Organizar & 9 & 21 & & & & \\
\hline & Prioridad & 5 & 10 & & & & \\
\hline & Importantes & 3 & 5 & & \multirow{8}{*}{ Urgentes } & \multirow{8}{*}{5} & \multirow{8}{*}{5} \\
\hline & Listas & 3 & 5 & & & & \\
\hline & Planificar & 4 & 8 & & & & \\
\hline & Horario & 7 & 17 & & & & \\
\hline & Distribuir & 3 & 6 & & & & \\
\hline & Actividades & 8 & 21 & & & & \\
\hline & Semana & 4 & 9 & & & & \\
\hline & Diario & 4 & 9 & & & & \\
\hline
\end{tabular}


Tabla 5: continuación

\begin{tabular}{|c|c|c|c|c|c|c|c|}
\hline \multicolumn{4}{|c|}{ Tiene antecedentes de estudios universitarios } & \multicolumn{4}{|c|}{ No tiene antecedentes de estudios universitarios } \\
\hline Dimensión & Palabra & F. Interna & F. Global & Dimensión & Palabra & F. Interna & F. Global \\
\hline \multirow{4}{*}{$\begin{array}{l}\text { Herramientas de } \\
\text { apoyo para } \\
\text { administrar el tiempo }\end{array}$} & Agenda & 5 & 7 & \multirow{4}{*}{$\begin{array}{l}\text { Herramientas de } \\
\text { apoyo para } \\
\text { administrar el } \\
\text { tiempo }\end{array}$} & Calendario & 10 & 11 \\
\hline & Reloj & 2 & 2 & & Computador & 8 & 9 \\
\hline & Listas & 4 & 7 & & Annto & \multirow{2}{*}{4} & \multirow{2}{*}{4} \\
\hline & Planeador & 3 & 6 & & Antolo & & \\
\hline \multirow{4}{*}{$\begin{array}{l}\text { Elementos que tiene } \\
\text { en cuenta al formular } \\
\text { objetivos }\end{array}$} & Quiero & 3 & 3 & \multirow{4}{*}{$\begin{array}{l}\text { Elementos que } \\
\text { tiene en cuenta al } \\
\text { formular objetivos }\end{array}$} & Actividades & 6 & 6 \\
\hline & Entrega & 2 & 2 & & & \multirow{3}{*}{12} & \multirow{3}{*}{14} \\
\hline & Aprender & 2 & 2 & & Tiempo & & \\
\hline & Tareas & 2 & 3 & & & & \\
\hline \multirow{6}{*}{$\begin{array}{l}\text { Estrategias de } \\
\text { priorización }\end{array}$} & Tareas & 6 & 8 & \multirow{6}{*}{$\begin{array}{l}\text { Estrategias de } \\
\text { priorización }\end{array}$} & Actividades & 25 & 28 \\
\hline & Concentración & 3 & 3 & & Trabajos & 11 & 12 \\
\hline & Acabar & 3 & 3 & & Complejas & 5 & 5 \\
\hline & Dificultad & 6 & 11 & & Horarios & 8 & 9 \\
\hline & Agenda & 2 & 2 & & \multirow{2}{*}{ Urgencia } & \multirow{2}{*}{4} & \multirow{2}{*}{4} \\
\hline & Suficiente & 2 & 2 & & & & \\
\hline
\end{tabular}

El grupo con antecedentes universitarios reportó mayor cantidad de estrategias para la planificación y uso del tiempo. Mostró acciones orientadas al uso de franjas de trabajo en la mañana o en la noche, planear periodicidad de actividades por semana o día. También hizo énfasis en el uso de horarios, listas y agendas y buscan organizar, distribuir y planificar las actividades de acuerdo con la importancia y la prioridad. Las herramientas de apoyo de grupo, corresponden a agendas, el reloj, listas y planeadores. Los objetivos de estudio se caracterizan por estar orientados a lo que se desea obtener (vocablo "quiero"), las entregas y tareas y lo que se desea aprender. Las estrategias de priorización se realizan con base en criterios orientados a las demandas de tareas a realizar, la dificultad, la suficiencia de tiempo y la utilización de agenda.

\section{Formulación de Objetivos de Estudio}

Los resultados no mostraron diferencias significativas para la sub-escala de planificación $(z=-0.740, p=$ 0.459 ) (ver tabla 1) teniendo en cuenta si se formulan o no objetivos de estudio. Los análisis textuales (tabla 6) mostraron que los estudiantes que formulan objetivos implementan estrategias de planificación teniendo en cuenta el tiempo, las demandas pendientes y lo que tienen la posibilidad de realizar (vocablos "puedo" y "poder"). Estas estrategias se orientan al establecimiento de límites, incluir el tiempo de descanso, el uso de notas y la organización de diarios. Este grupo se apoya en el uso de herramientas como agendas, aplicaciones, anotaciones, internet y alarmas. La priorización de actividades la realizan a través de métodos de organización con base en criterios de urgencia, de tiempo y buscando la finalización de la tarea (vocablo acabar) y disponer de descanso.

Los estudiantes que no reportaron formular objetivos de estudio implementan estrategias orientadas a atender las necesidades, las actividades urgentes y se caracterizan por considerar franjas de estudio principalmente en la noche. Se apoyan en herramientas como el celular, cronogramas, cuadernos, notas, lecturas, software, guías y calendarios. Priorizan con base en la facilidad de la tarea, la carga de la actividad y las características de la tarea. Reportaron hacer uso de planeadores para priorizar.

Tabla 6: Método especificidades, formulación de objetivos de estudio

\begin{tabular}{|c|c|c|c|c|c|c|c|}
\hline \multicolumn{4}{|c|}{ Formula objetivos de estudio } & \multicolumn{4}{|c|}{ No formula objetivos de estudio } \\
\hline Dimensión & Palabra & F. Interna & F. Global & Dimensión & Palabra & F. Interna & F. Global \\
\hline \multirow{8}{*}{$\begin{array}{l}\text { Estrategias de } \\
\text { planificación }\end{array}$} & Tiempo & 27 & 30 & \multirow{6}{*}{$\begin{array}{l}\text { Estrategias de } \\
\text { planificación }\end{array}$} & Noche & 3 & 3 \\
\hline & Descanso & 4 & 4 & & Urgentes & 4 & 5 \\
\hline & Notas & 3 & 3 & & Celular & 2 & 2 \\
\hline & Pendiente & 3 & 3 & & Espacio & 2 & 2 \\
\hline & Limite & 3 & 3 & & Especificar & 2 & 2 \\
\hline & Pueda & 3 & 3 & & Necesidad & 3 & 4 \\
\hline & Poder & 3 & 3 & \multirow{8}{*}{$\begin{array}{l}\text { Herramientas } \\
\text { de apoyo para } \\
\text { administrar el } \\
\text { tiempo }\end{array}$} & Cronograma & 4 & 5 \\
\hline & Diario & 7 & 9 & & Cuaderno & 2 & 2 \\
\hline \multirow{6}{*}{$\begin{array}{l}\text { Herramientas de apoyo } \\
\text { para administrar el } \\
\text { tiempo }\end{array}$} & Agenda & 7 & 7 & & Notas & 2 & 2 \\
\hline & Aplicaciones & 4 & 4 & & Leer & 2 & 2 \\
\hline & Anoto & 4 & 4 & & Excel & 3 & 4 \\
\hline & Internet & 2 & 3 & & Cronometro & 2 & 3 \\
\hline & \multirow{2}{*}{ Alarmas } & \multirow{2}{*}{2} & \multirow{2}{*}{3} & & Guías & 2 & 3 \\
\hline & & & & & Calendario & 5 & 11 \\
\hline
\end{tabular}


Tabla 6: continuación

\begin{tabular}{|c|c|c|c|c|c|c|c|}
\hline Dimensión & Palabra & F. Interna & F. Global & Dimensión & Palabra & F. Interna & F. Global \\
\hline \multirow{6}{*}{$\begin{array}{l}\text { Estrategias de } \\
\text { priorización }\end{array}$} & Organizo & 13 & 17 & \multirow{6}{*}{$\begin{array}{l}\text { Estrategias de } \\
\text { priorización }\end{array}$} & \multirow{2}{*}{ Fáciles } & \multirow{2}{*}{7} & \multirow{2}{*}{10} \\
\hline & Urgencia & 4 & 4 & & & & \\
\hline & Descanso & 4 & 4 & & Carga & 3 & 3 \\
\hline & Tiempo & 23 & 33 & & Sencillo & 2 & 2 \\
\hline & Concentración & 3 & 3 & & Planeador & 2 & 2 \\
\hline & Acabar & 3 & 3 & & Tareas & 5 & 8 \\
\hline
\end{tabular}

\section{Desempeño Académico}

No se identificaron diferencias significativas entre en el promedio acumulado $(z=-0.757, p=0.449)$ y del periodo anterior $(z=-0.740, p=0.459)$ respecto a los puntajes de la escala de planificación (ver tabla 1$)$. Los análisis textuales (ver tabla 7), mostraron que los estudiantes del grupo mayor desempeño, implementan de estrategias orientadas al uso de diarios, la planificación y la implementación de listas de actividades. Utilizan herramientas como horarios, calendarios y aplicaciones. Los objetivos de estudio los formulan teniendo en cuenta el tiempo, las actividades que deben cumplir, los deberes y las tareas. Con relación a las actividades de priorización no se observaron estrategias en este grupo que mostraran diferencias con respecto al subcorpus del grupo de menor rendimiento.

Tabla 7: Método especificidades, promedio académico

\begin{tabular}{|c|c|c|c|c|c|c|c|}
\hline \multicolumn{4}{|c|}{ Promedio acumulado grupo bajo } & \multicolumn{4}{|c|}{ Promedio acumulado grupo alto } \\
\hline Dimensión & Palabra & F. Interna & F. Global & Dimensión & Palabra & F. Interna & F. Global \\
\hline \multirow{10}{*}{$\begin{array}{l}\text { Estrategias de } \\
\text { planificación }\end{array}$} & Investigar & 3 & 3 & \multirow{10}{*}{$\begin{array}{l}\text { Estrategias } \\
\text { de } \\
\text { planificación }\end{array}$} & \multirow{3}{*}{ Diario } & \multirow{3}{*}{9} & \multirow{3}{*}{9} \\
\hline & Organizar & 10 & 21 & & & & \\
\hline & Leer & 4 & 6 & & & & \\
\hline & Horario & 8 & 17 & & \multirow{4}{*}{ Planificar } & \multirow{4}{*}{8} & \multirow{4}{*}{8} \\
\hline & Levantarme & 2 & 2 & & & & \\
\hline & Reuniones & 2 & 2 & & & & \\
\hline & Escribir & 2 & 3 & & & & \\
\hline & Clases & 2 & 3 & & \multirow{3}{*}{ Listas } & \multirow{3}{*}{5} & \multirow{3}{*}{5} \\
\hline & Cronograma & 2 & 3 & & & & \\
\hline & Estudiar & 4 & 9 & & & & \\
\hline \multirow{8}{*}{$\begin{array}{l}\text { Herramientas de } \\
\text { apoyo para } \\
\text { administrar el tiempo }\end{array}$} & Libros & 5 & 7 & \multirow{8}{*}{$\begin{array}{l}\text { Herramientas } \\
\text { de apoyo } \\
\text { para } \\
\text { administrar el } \\
\text { tiempo }\end{array}$} & \multirow{4}{*}{ Horarios } & \multirow{4}{*}{5} & \multirow{4}{*}{5} \\
\hline & Ninguna & 3 & 4 & & & & \\
\hline & Reloj & 2 & 2 & & & & \\
\hline & Notas & 2 & 2 & & & & \\
\hline & Lecturas & 2 & 2 & & \multirow{4}{*}{ Aplicaciones } & \multirow{4}{*}{4} & \multirow{4}{*}{4} \\
\hline & Computador & 5 & 9 & & & & \\
\hline & Guías & 2 & 3 & & & & \\
\hline & Internet & 2 & 3 & & & & \\
\hline \multirow{4}{*}{$\begin{array}{l}\text { Elementos que tiene } \\
\text { en cuenta al } \\
\text { formular objetivos }\end{array}$} & Metas & 3 & 4 & \multirow{8}{*}{$\begin{array}{l}\text { Elementos } \\
\text { que tiene en } \\
\text { cuenta al } \\
\text { formular } \\
\text { objetivos }\end{array}$} & Cumplir & 6 & 6 \\
\hline & Materia & 2 & 2 & & Debo & 4 & 4 \\
\hline & Tema & 4 & 8 & & & & \\
\hline & Poder & 2 & 3 & & Realızar & 7 & 8 \\
\hline \multirow{4}{*}{$\begin{array}{l}\text { Estrategias de } \\
\text { priorización }\end{array}$} & Dificultad & 6 & 11 & & & & \\
\hline & Tiempo & 14 & 33 & & Tiempo & 11 & 14 \\
\hline & Horarios & 5 & 9 & & & & \\
\hline & Actividades & 12 & 28 & & Tareas & 3 & 3 \\
\hline Promedio & semestre ante & rgrupo bajc & & Prome & dio semestre a & erior grupo & \\
\hline & Horario & 8 & 17 & & & & \\
\hline Estrategias de & Necesidad & 3 & 4 & Estrategias & & & \\
\hline planificación & Trabajos & 6 & 14 & & Planıtıcar & 8 & 8 \\
\hline & Organizar & 8 & 21 & & & & \\
\hline & Computador & 6 & 9 & & listas & 5 & 5 \\
\hline & Libros & 5 & 7 & & LIstas & 5 & 5 \\
\hline Herramientas de & Reloj & 2 & 2 & Herramientas & & & \\
\hline $\begin{array}{l}\text { apoyo para } \\
\text { administrar el tiemno }\end{array}$ & Lecturas & 2 & 2 & de apoyo & Horarios & 5 & 5 \\
\hline & Guías & 2 & 3 & para & & & \\
\hline & Apuntes & 3 & 6 & administrar el & Aplicaciones & 4 & 4 \\
\hline Elementos que tiene & Materia & 2 & 2 & tiempo & & & \\
\hline en cuenta al & Poder & 2 & 3 & & & & \\
\hline formular objetivos & Pendientes & 2 & 4 & & Calendario & 9 & 11 \\
\hline
\end{tabular}


Tabla 7: continuación

\begin{tabular}{|c|c|c|c|c|c|c|c|}
\hline \multicolumn{4}{|c|}{ Promedio semestre anterior grupo bajo } & \multicolumn{4}{|c|}{ Promedio semestre anterior grupo alto } \\
\hline Dimensión & Palabra & Dimensión & Palabra & Dimensión & Palabra & Dimensión & Palabra \\
\hline \multirow{3}{*}{$\begin{array}{l}\text { Estrategias de } \\
\text { priorización }\end{array}$} & Materia & 2 & 2 & \multirow{3}{*}{$\begin{array}{c}\text { Elementos que } \\
\text { tiene en cuenta al } \\
\text { formular objetivos }\end{array}$} & \multirow{3}{*}{ Cumplir } & \multirow{3}{*}{6} & \multirow{3}{*}{6} \\
\hline & Poder & 2 & 3 & & & & \\
\hline & Pendientes & 2 & 4 & & & & \\
\hline
\end{tabular}

El grupo de menor rendimiento académico reportó estrategias para la planificación orientadas a la organización, uso de horarios, lecturas y cronogramas y tener en cuenta las necesidades académicas. Las herramientas en las que se apoyan corresponden a libros, reloj, notas o apuntes, lecturas, computador, guías, internet o no reportaron uso de apoyos. Los objetivos de estudio los formulan teniendo en cuenta las materias que presentan deberes o actividades pendientes y el tema al que se orienta la tarea y lo que tiene la posibilidad de realizar. La priorización la orientan al establecimiento de horarios, las actividades, el tiempo, la dificultad de la actividad, la materia a trabajar y las actividades pendientes.

\section{Desempeño Académico y Habilidades de Planificación}

EI ACM aplicado para caracterizar las estrategias de planificación, uso de herramientas, formulación de objetivos y acciones de priorización del tiempo de acuerdo con el desempeño académico y el resultado de la escala de planificación, se presenta en un plano factorial de dos ejes que muestran el porcentaje de varianza explicada del corpus textual obtenido en las preguntas abiertas incluidas en los formularios. La tabla 8 muestra el porcentaje de varianza explicada para cada uno de los análisis realizados.

Tabla 8: Resultados plano factorial ACM

\begin{tabular}{|c|c|c|c|c|c|c|c|c|}
\hline \multirow{2}{*}{ Eje } & \multicolumn{2}{|c|}{$\begin{array}{c}\text { Estrategias de } \\
\text { planificación }\end{array}$} & \multicolumn{2}{c|}{$\begin{array}{c}\text { Herramientas de apoyo a } \\
\text { la planificación }\end{array}$} & \multicolumn{2}{c|}{$\begin{array}{c}\text { Elementos para la } \\
\text { formulación de objetivos }\end{array}$} & \multicolumn{2}{c|}{ Estrategias de priorización } \\
\cline { 2 - 9 } & Var Expl. & $\begin{array}{c}\text { Ac. Var. } \\
\text { Expl. }\end{array}$ & Var Expl & $\begin{array}{c}\text { Ac. Var. } \\
\text { Expl. }\end{array}$ & $\begin{array}{c}\text { Var } \\
\text { Expl. }\end{array}$ & Ac. Var. Expl. & Var Expl. & Ac. Var. Expl. \\
\hline 1 & $\% 76.39$ & $\% 76.39$ & $\% 62.5$ & $\% 62.5$ & $\% 75.42$ & $\% 75.42$ & $\% 57.70$ & $\% 57.70$ \\
\hline 2 & $\% 22.20$ & $\% 98.59$ & $\% 34.1$ & $\% 96.7$ & $\% 20.33$ & $\% 95.75$ & $\% 37.54$ & $\% 95.24$ \\
\hline
\end{tabular}

Los resultados respecto a las estrategias de planificación evaluados en el grupo de estudiantes participantes, mostraron que los dos ejes del primer plano factorial agrupan el $98 \%$ de la varianza del corpus (ver tabla 8). La figura 1 muestra los resultados correspondientes a las contribuciones de los términos para cada eje. El eje uno explica un $76.39 \%$ de la varianza del corpus textual y corresponde al desempeño académico reportado a través del promedio académico. El eje dos que explica un $22.20 \%$ de la varianza corresponde al puntaje en la sub-escala de planificación del MAI.

La figura 1 presenta los términos reportados por los participantes respecto a las estrategias de planificación de acuerdo con el promedio académico y la sub-escala de planificación. Se presenta la información por cuadrante, el superior izquierdo corresponde a las estrategias de los estudiantes que reportaron rendimiento menor y puntajes en la escala de planificación bajo, se observan estrategias que se orientan la implementación de reuniones, cronogramas, horarios, lectura y escritura. El superior derecho corresponde a las estrategias de los estudiantes del grupo de rendimiento alto y puntajes de planificación bajos, se reportan estrategias orientadas a planificar de acuerdo con las características de las materias, las fechas de entrega, horarios de trabajo (día/noche) y el uso de listas. El inferior izquierdo corresponde a los participantes que reportaron rendimiento bajo y mayor puntaje en la escala de planificación. Se observan acciones orientadas a la planificación con base en las clases a tomar y búsqueda de organización, apoyo con tutorías, madrugar - levantarse más temprano. Finalmente, en el inferior derecho se encuentran las estrategias de los estudiantes de desempeño y puntajes en la escala de planificación altos. Se evidenciaron acciones asociadas a la priorización, la planificación, la organización diaria de actividades de estudio, el uso de agendas y la consideración del tiempo de descanso durante el ejercicio de las actividades académicas realizadas en los espacios de estudio fuera de clase.

Algunos casos que muestran las estrategias de planificación implementadas por los estudiantes que participaron en el estudio para cada plano factorial corresponden a las siguientes. 1) Rendimiento Bajo Escala Planificación Alto: "Caso 39: Me propongo una hora para reunirme a hacer trabajos grupales y el resto del tiempo hago los individuales"; 2) Rendimiento Alto - Escala Planificación Bajo: "Caso 43: Ocasionalmente suelo dar prioridad a las materias cuyos trabajos tienen mayor dificultad, de modo que reparto la carga en la 
semana de lo fácil o lo más difícil"; 3) Rendimiento Bajo - Planificación Alto: "Caso 29: Establezco mis tiempos y organizo cada uno de ellos para poder tener un orden en cada tarea"; 4) Rendimiento Alto - Planificación Alto:" Caso 1: organizo por prioridades de actividad, sin mezclarlo con mi tiempo de ocio".

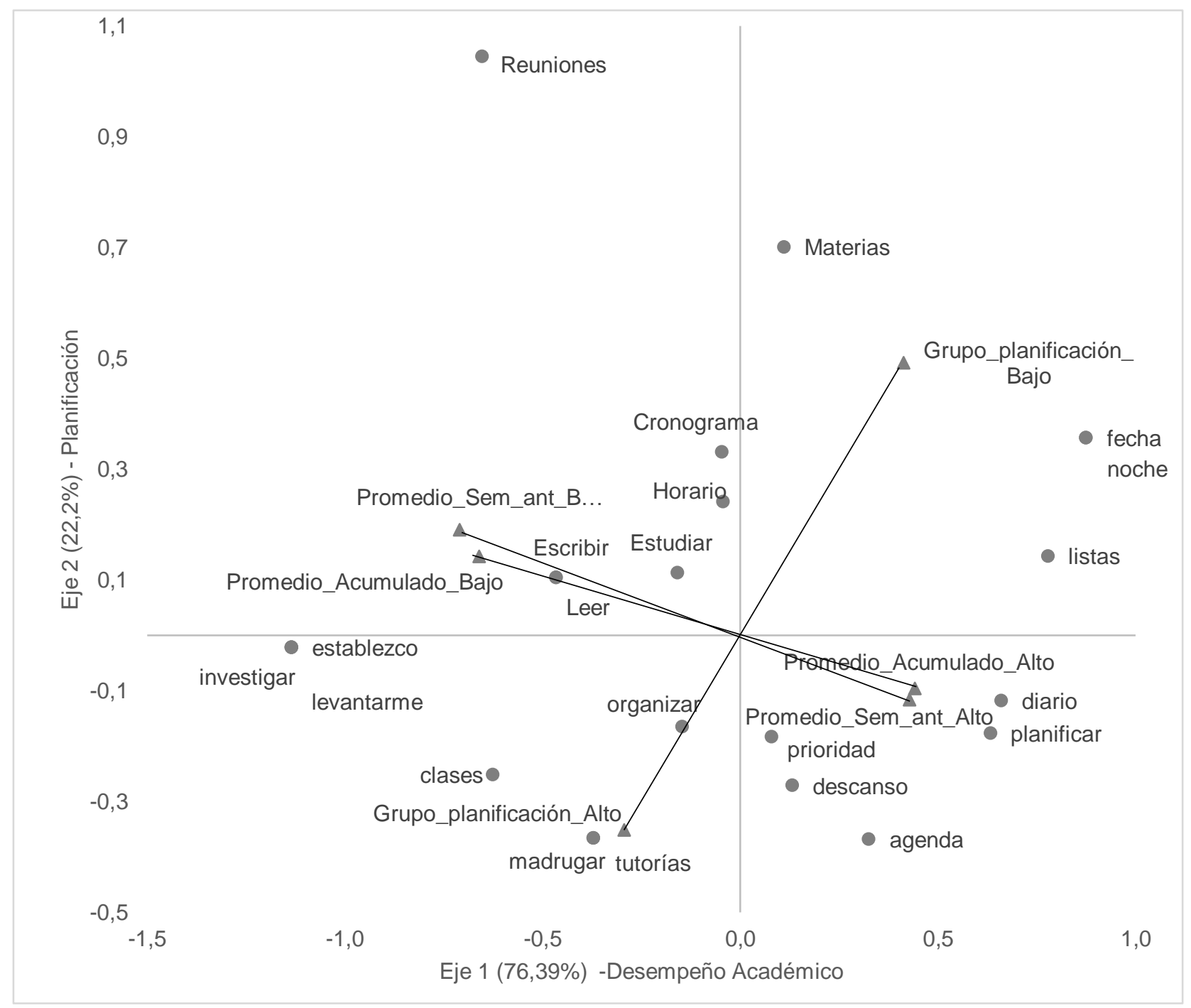

Fig. 1: Plano factorial estrategias de planificación del tiempo

Respecto a las herramientas de apoyo para la planificación del tiempo de estudios, la figura 2 muestra el plano factorial que agrupa el $96.7 \%$ de la varianza del corpus textual (ver tabla 8 ). El eje uno (62\% de la varianza), corresponde al desempeño académico reportado por los estudiantes y el eje dos (34.1\% de la varianza) al puntaje de la escala de planificación del MAl. Se presentan las herramientas de apoyo por cuadrante. El superior izquierdo muestra las herramientas que reportaron los estudiantes con rendimiento y puntajes en la escala de planificación altos. Se observa uso del teléfono celular, notas, horarios, calendarios. El superior derecho corresponde a los estudiantes que reportaron rendimiento bajo y mayores puntajes en la escala de planificación. Este grupo suele apoyarse de herramientas como apuntes, guías, lecturas, libros o computadores. El inferior izquierdo corresponde a los estudiantes que indicaron tener alto desempeño y menor puntaje en la escala de planificación. Se observó uso de menor cantidad de herramientas de apoyo dentro de las que se encuentra el uso de agendas, aplicaciones y recordatorios. Finalmente, el inferior derecho muestra las herramientas que reportaron los estudiantes con menor desempeño y puntaje en la escala de planificación. Se observa el uso de notas, internet, el reloj e incluso declaran no usar apoyos.

Algunos casos representativos son 1) Rendimiento Alto - Planificación Alto: "Caso 9: una libreta donde anoto horarios, tareas a hacer y voy chuleando al terminar cada una de ellas"; 2) Rendimiento Bajo - Escala Planificación Alto: "Caso 29: realizo apuntes en un planeador donde llevo anotación de cada una de mis tareas a realizar"; 3) Rendimiento Alto - Escala Planificación Bajo: "Caso 18: utilizo una agenda en donde programo las actividades a entregar durante la semana."; 4) Rendimiento Bajo - Planificación Bajo: "Caso 31: notas para estar pendiente de lo que debo hacer con más urgencia". 
La figura 3 presenta el plano factorial relativo a los elementos que tienen en cuenta los estudiantes para la formulación de objetivos de estudio. El plano factorial presenta dos ejes que agrupan el $95.75 \%$ de la varianza del corpus textual (ver tabla 8). El eje uno corresponde al promedio académico (75,4\% de la varianza) y el eje dos corresponde al puntaje en la escala de planificación (20.33\% de la varianza). La información se presenta por cuadrante, en el superior izquierdo se presentan los elementos que tienen los estudiantes con menor promedio académico y puntaje en la escala de planificación. Estos estudiantes suelen tener en cuenta las materias que cursan, metas formuladas y asuntos pendientes. En el superior derecho, los elementos que tienen en cuenta los estudiantes con mayor rendimiento académico y bajo puntaje en la escala de planificación MAI. Se observan elementos relacionados con las horas dedicadas al trabajo académico, lo que el estudiante está en posibilidad de realizar y el tiempo que dispone. En inferior izquierdo, se presentan los aspectos que tienen en cuenta los estudiantes que reportaron menor desempeño académico y puntajes de planificación alto. Se observaron criterios relacionados con la posibilidad que tiene el estudiante de cumplir o finalizar los deberes, actividades asociadas a días de trabajo académico y organización en el desarrollo de actividades. El inferior derecho muestra los factores que tienen presente los estudiantes que reportaron mayor desempeño y puntaje en la escala de planificación. Se observaron elementos orientados al cumplimiento de plazos, intereses y actividades académicas.

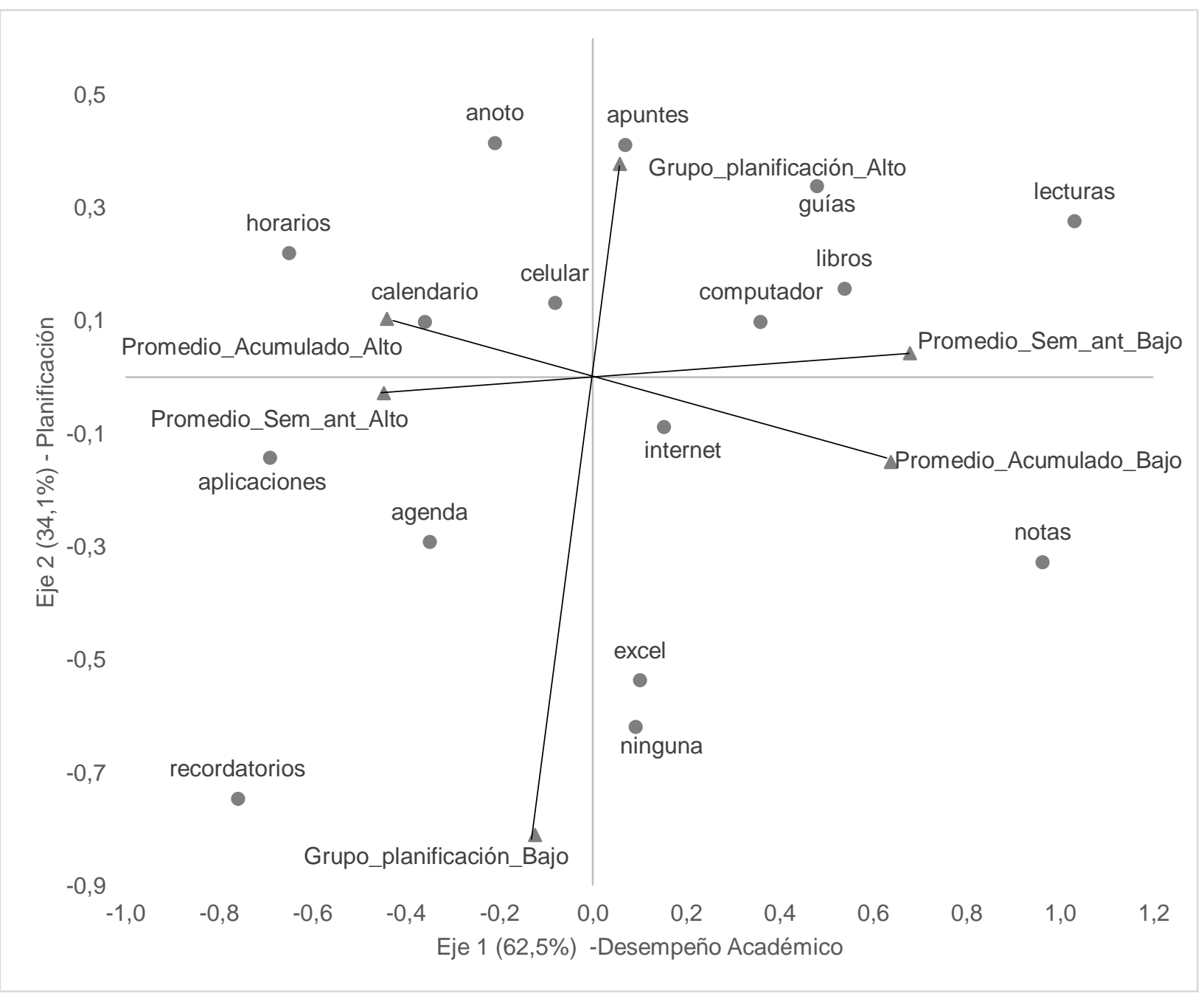

Fig. 2: Plano factorial herramientas de apoyo a la planificación del tiempo

Algunos casos representativos son 1) Rendimiento Bajo - Planificación Bajo: "Caso 72: estudiar sobre la materia que voy a ver el día anterior y revisar el ava (aula virtual) para ver que tengo pendiente"; 2) Rendimiento Alto - Escala Planificación Bajo: "Caso 76: "tener horas específicas para concentrarme en las cosas de la universidad"; 3) Rendimiento Bajo - Escala Planificación Alto:" Caso 44: Debo terminar de leer o de realizar un trabajo en cierta hora específica"; 4) Rendimiento Alto - Planificación Alto:" Caso 8: destinar tiempos a cada actividad y plazos de realización de los mismos". 
La figura 4 muestra las estrategias de priorización reportadas por los estudiantes de acuerdo con el rendimiento reportado y el resultado de la escala de planificación MAl. El plano factorial muestra dos ejes que agrupan el $95.2 \%$ de la varianza del corpus, el eje uno corresponde al desempeño académico $(57.7 \%$ de la varianza) y el eje dos corresponde al resultado de la escala de planificación (37.5\% de la varianza). La información se presenta por cuadrante. En el superior izquierdo las estrategias de priorización de los estudiantes con mayor desempeño y puntajes en la escala de planificación. Se observa uso de estrategias orientas a la finalización de las actividades pendientes, favorecer la concentración y el descanso. En el cuadrante superior derecho se presentan las estrategias de los estudiantes con menor desempeño y mayores puntajes en la escala de planificación. Se observan estrategias orientadas a la dificultad o complejidad de las tareas principalmente. En el inferior izquierdo se presentan las estrategias de priorización de los estudiantes que reportaron mayor desempeño y menor puntaje en la escala de planificación. Se observa priorización orientada a la distribución de tiempos y actividades, a la entrega de actividades pendientes, y al uso del tiempo libre para resolver actividades con mayor prioridad. Finalmente, en el inferior derecho se presentan las estrategias los estudiantes con menor desempeño y puntaje en la escala de planificación. Se observan priorización de acuerdo con las características de las actividades, uso de horarios y organización secuencial dando énfasis con las actividades que programan para iniciar.

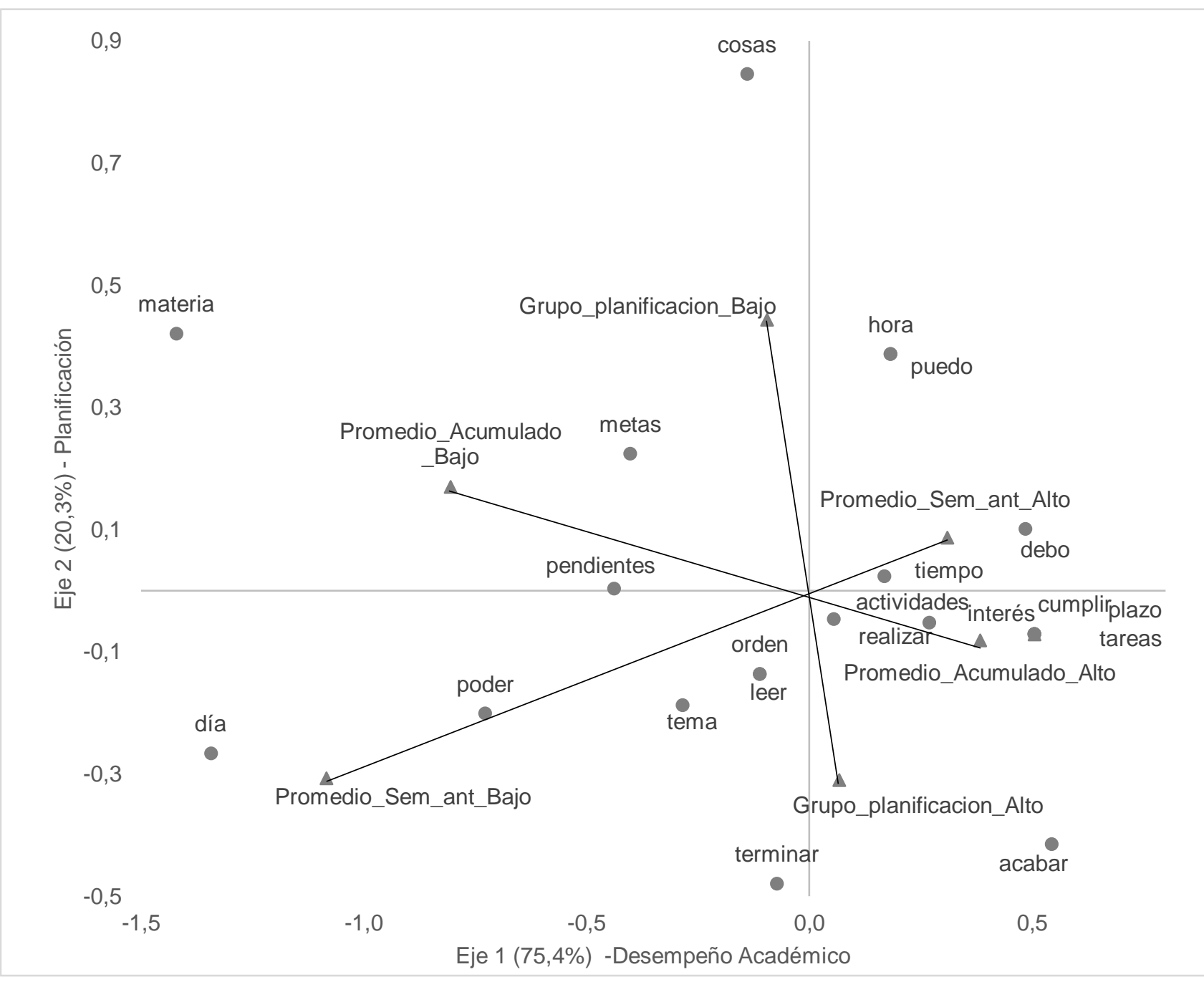

Fig. 3: Plano factorial elementos tenidos en cuenta en la formulación de objetivos de estudio

Algunos casos representativos son (a) Rendimiento Alto - Planificación Alto: "Caso 35: Suelo seguir de largo hasta que acabe lo más complicado. Duermo poco pero después de tener todo listo descanso lo suficiente."; (b) Rendimiento Bajo - Escala Planificación Alto: "Caso 10: Comienzo con lo que considero sencillo y termino con lo complejo"; (c) Rendimiento Alto - Planificación Bajo: "Caso 27: Siempre por orden de entrega, distribuyo los tiempos en horas libres, las que me quedan después de clase en la semana y el día sábado"; (d) Rendimiento Bajo - Escala Planificación Bajo: "Caso5: "Comienzo con la que se más haga más fácil y la más corta." 


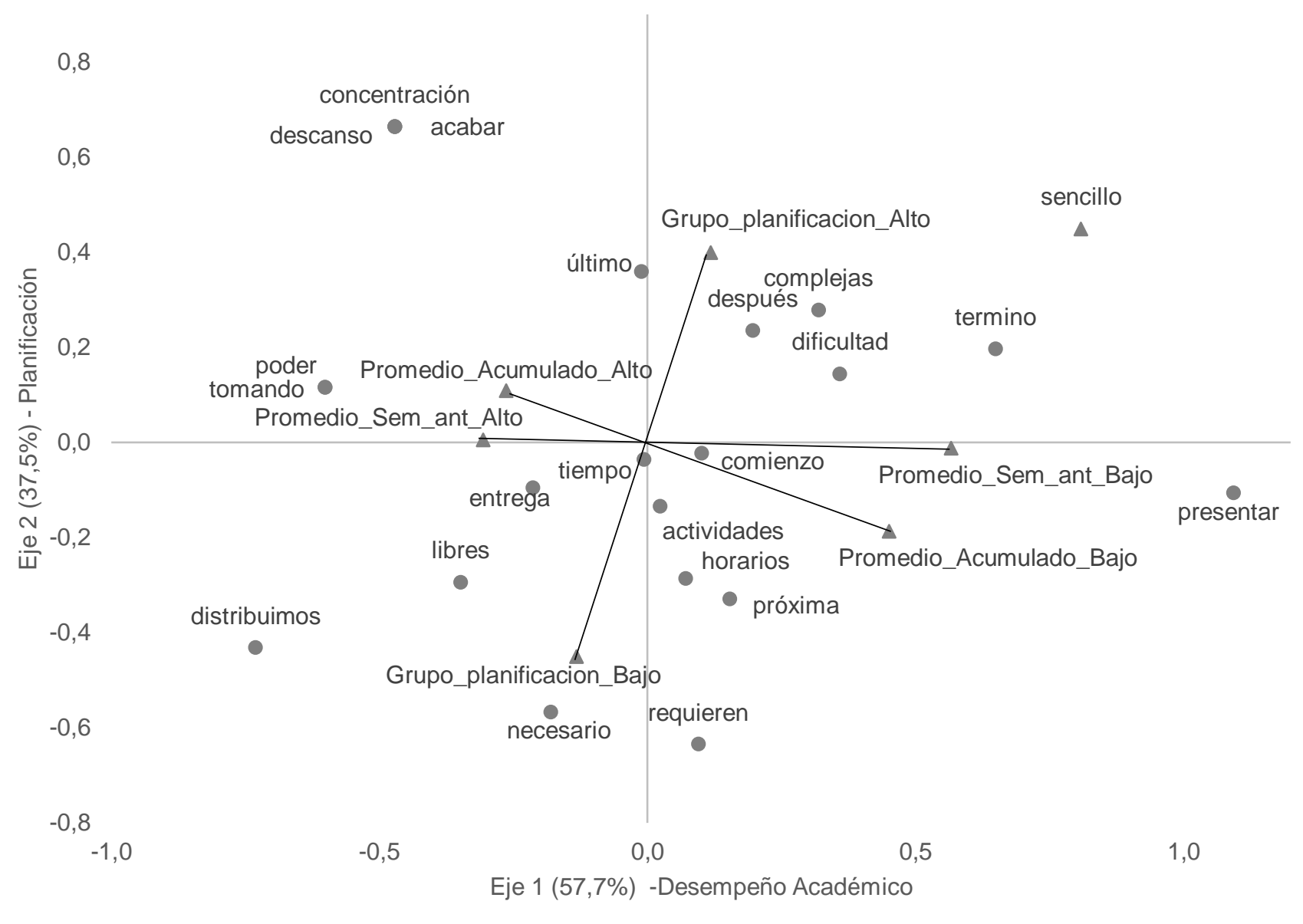

Fig. 4: Plano factorial estrategias de priorización

\section{DISCUSIÓN}

Los resultados obtenidos muestran que la evaluación de las estrategias para la planificación a través de la sub-escala del MAI no tuvo diferencias significativas de acuerdo con el sexo. Lo anterior se muestra acorde con los resultados obtenidos por Garzón-Umerenkova y Gil-Flores (2017) y Broc (2011) quienes no replicaron resultados de esta variable en conductas de procrastinación y gestión del tiempo. Sin embargo, los resultados textuales reportados por los estudiantes muestran en las mujeres mayor tendencia a la organización y al uso de estrategias para la priorización con base en fechas, tareas por realizar o tiempo disponible, lo cual también fue referido por Elvira-Valdés y Pujol (2012) y Durán-Aponte y Pujol (2013) quienes reportan mayor uso de estrategias para la gestión del tiempo en las mujeres. A diferencia de estos estudios, para este caso, los hombres reportaron mayor uso apoyos o herramientas de apoyo.

No se encontraron diferencias significativas respecto a las habilidades de planificación evaluadas a través de la escala del MAI respecto a la jornada de estudio y el compromiso horario por actividad laboral. Los resultados no son correspondientes con los reportado por Garzón-Umerenkova y Gil-Flores (2017), quienes encontraron que los estudiantes con actividad laboral tienden a tener mayor riesgo de procrastinación debido a que destinan más de media jornada a la actividad laboral. Sin embargo y en consideración lo propuesto por Robotham (2012) que sugiere que los estudiantes que destinan tiempo parcial al estudio requieren de mayor habilidad para la gestión del tiempo, los análisis textuales mostraron que los estudiantes que toman sus clases en la noche, quienes en un $73 \%$ tienen compromiso horario por actividad laboral, expresaron mayor variabilidad en las estrategias para planificar. Según sus respuestas, lo hacen con base en el uso de notas o agendas; buscan organizar y planificar de acuerdo con el tipo de tarea y la importancia de la misma y suelen administrar los tiempos disponibles en franjas regulares diarias o semanales y en horarios como las madrugadas o noches. También reportaron contemplar apoyos como tutorías. Estos estudiantes priorizan con base en las entregas pendientes, el tiempo disponible y una organización de actividades. Las herramientas que usan de apoyo corresponden a agendas, notas y planeadores o, no se apoyan en ninguna herramienta. Un $66 \%$ de los estudiantes que estudian en la noche reporta formular objetivos de estudio y un $53 \%$ de los que labora los formula. Estos objetivos, los formulan con base en la distribución de las actividades pendientes, en la planificación, distribución y entrega oportuna de actividades. 
Por otro lado, los estudiantes de la jornada diurna que en un $27 \%$ tienen actividad, mostraron menor variabilidad en las estrategias implementadas para la planificación. Estas se orientan a la priorización con base en la dificultad de la tarea, buscan dar alcance a las actividades de acuerdo con el tiempo que disponen y se orientan a resolver las actividades pendientes. Un 54\% de los estudiantes que estudian en el día, reportó formular objetivos de estudio y un $61 \%$ de los que no laboran los formula. Estos objetivos los formulan teniendo en cuenta la complejidad de la tarea, distribuir el tiempo y dar respuesta a los pendientes de acuerdo con sus posibilidades. Las estrategias para priorizar se enfocan en las características de las labores a realizar, distribuir el tiempo y dar tiempo para el descanso. Por otro lado, reportaron hacer uso de diversas herramientas de apoyo como el celular, listas de trabajo o trabajo en grupo.

La existencia de experiencia académica previa en la universidad tampoco mostró diferencias significativas en la escala de planificación (MAI). Estos resultados se muestran acorde con lo encontrado por Crede y Niehorster (2012) que no encontraron relaciones entre experiencias universitarias previas y ajuste académico, definido como el grado en que existe adaptación de los estudiantes para cumplir las demandas académicas. Sin embargo, los estudiantes que han realizado estudios universitarios previos reportaron mayor variabilidad en las estrategias de planificación como son uso de horarios, listas o agendas que permitan organización de acuerdo con la frecuencia de las actividades (semanal, diaria) y con el objetivo de planificar, distribuir, organizar y priorizar. $57 \%$ suelen formular objetivos de estudio y lo hacen teniendo en cuenta las características de las actividades. Reportan priorizar de acuerdo con las entregas pendientes, la dificultad, la disposición del tiempo y la priorización e indicaron apoyarse en planeadores, agendas y listas.

La contrastación entre las estrategias que implementan los estudiantes que suelen implementar objetivos de estudio frente a los que no, no mostró diferencias respecto a la escala de planificación (MAI). No obstante, se observó que $59 \%$ formulan objetivos de estudio. Estos mostraron mayor diversidad de estrategias las cuales tienen en cuenta demandas, capacidades, alcances y tiempo para descanso. También se observó en este grupo mayor variabilidad en las estrategias para priorizar actividades buscando organizar con base en la urgencia de la actividad, los tiempos disponibles, el alcance los trabajos y considerando tiempo para descanso. Estos estudiantes reportaron apoyarse en agendas, aplicaciones, notas o alarmas. Los estudiantes que no reportaron usar objetivos de estudio mostraron menor variabilidad de estrategias. Estas principalmente se centran en las demandas, prioridades para organizar el tiempo. Este grupo reportó priorizar actividades con base en la dificultad y el tipo de tarea y reportaron mayor uso de apoyos como celular, cronogramas, notas, lecturas o software.

Respecto al desempeño académico, no se observaron diferencias en la escala de planificación entre los estudiantes. Lo anterior es acorde con los resultados encontrados en estudios previos que no reportaron diferencias significativas entre los estudiantes con alto o bajo desempeño y una correlación baja entre la gestión del tiempo y el desempeño (De la Barrera et al., 2008; Garzón Umerenkova y Flores, 2018). Al respecto, los análisis textuales mostraron que las estrategias de los estudiantes con mayor rendimiento se asocian a planificar, priorizar y organizar franjas de estudio en el día o en la noche. El ACM mostró que, de este grupo, los estudiantes que reportaron mayor puntaje en la escala del MAl indicaron uso de estrategias orientadas a planificar y dar prioridad, usar agendas y considerar tiempo de descanso. Los que reportaron menores puntajes en el MAl, implementan acciones orientadas por el tipo de actividad, las fechas de entrega, la implementación de franjas de estudio y uso de listas.

Respecto a los estudiantes con menor desempeño, se observa uso de estrategias dirigidas principalmente a organizar a través del uso de horarios y cronogramas que permitan dar respuesta a las necesidades. El ACM mostró que, los estudiantes que reportaron mayores puntajes en la escala MAl, mostraron programar reuniones, organizar con base en cronogramas y horarios. Los que reportaron menor puntaje en la escala, indicaron planificar de acuerdo con las asignaturas, buscar apoyos como tutorías y estudiar en espacios determinados como la madrugada. Los resultados respecto a las diferencias en el desempeño en los estadísticos de Mann-Whitney y las diferencias de los resultados textuales, pueden estar relacionados con un conocimiento de las estrategias, pero un desconocimiento de la aplicación de mismas (Pérez et al., 2013)

Respecto a las estrategias de priorización, los resultados del ACM mostraron que, para los estudiantes con mayor desempeño, los mayores puntajes en la escala MAI suelen relacionarse con estrategias de priorización orientadas a las actividades que los estudiantes deben terminar, a facilitar la concentración y considerar tiempo de descanso. Por otro lado, menores puntajes en la escala muestran priorizar a partir del uso del tiempo libre y resolver actividades más urgentes. Por otro lado, los estudiantes con menor desempeño y mayores resultados en la escala de planificación mostraron priorizar con base en la dificultad de la tarea. Los que reportaron menores puntajes en la escala expresaron priorizar haciendo uso de secuencias horarias para decidir por qué actividad iniciar. Al respecto, Garzón Umerenkova y Flores (2018) sugieren que a pesar de que se identifican estrategias de planificación, es importante considerar si estas son efectivas en este grupo de estudiantes, para este caso no se observan respuestas orientadas al cumplimiento de las actividades programadas. 
Respecto a los objetivos de estudio, un 55\% de los estudiantes que reportaron mayor desempeño indicaron formular objetivos de estudio. Estos los establecen teniendo en cuenta el tiempo y las actividades por realizar. Los ACM mostraron que los mayores puntajes en la escala MAI, se relacionaron a objetivos caracterizados por el cumplimiento de plazos y a los intereses y actividades académicas. Es importante mencionar que la formulación de objetivos orientados a intereses es una característica mencionada por Zimmerman y Bandura (1994) respectiva a una percepción positiva de la regulación del proceso de aprendizaje. Por otro lado, los bajos puntajes en la escala mostraron objetivos de estudio en los que se tiene en cuenta la disponibilidad de tiempo y horas de trabajo académico.

De los estudiantes con menor desempeño un $62 \%$ indicaron formular objetivos de estudio. Estos los formulan orientados al cumplimiento de actividades para las asignaturas y las posibilidades que tiene para cumplir con los deberes. EI ACM mostró que, de este grupo, los estudiantes con mayor puntaje en la escala de planificación, indica tener en cuenta la posibilidad de realizar las actividades, la disposición de tiempo de trabajo y la organización. Los que tuvieron menor puntaje en la escala del MAl, reportaron objetivos orientados a las asignaturas que cursan, metas formuladas y actividades por realizar.

Las herramientas implementadas para dar apoyo a la planificación de los estudiantes con mayor desempeño son más variadas y corresponden a franjas horarias, calendario y aplicaciones. El ACM mostró que, dentro de este grupo, los mayores puntajes en la escala de planificación están relacionadas con diversas herramientas como horarios, notas y uso del teléfono celular. Los menores puntajes en la escala de planificación muestran uso de herramientas como recordatorios, agendas y aplicaciones. Estos resultados son correspondientes con lo reportado por Garzón Umerenkova y Flores (2018). Los estudiantes con menor desempeño indicaron uso de herramientas como libros, relojes, notas, guías o el computador. El ACM mostró que los mayores puntajes en el MAl están relacionados con el uso de apuntes, guías o lecturas. Por otro lado, los menores puntajes en esta escala se asociaron a las notas, computador o no usar ningún apoyo.

\section{CONCLUSIONES}

A pesar de no encontrar diferencias en la sub-escala de planificación, los análisis textuales sugieren diferencias en estrategias y herramientas de planificación de acuerdo con el sexo, antecedentes de estudios universitarios, formulación de objetivos de estudio, jornada y compromiso horario por actividad laboral. Respecto al desempeño académico, los análisis textuales y de correspondencia muestran diferencias entre estrategias de planificación, priorización, formulación de objetivos y herramientas de apoyo entre los estudiantes con mayor y menor promedio de estudio y puntaje en la sub-escala de planificación.

\section{AGRADECIMIENTOS}

Este estudio contó con el apoyo del Proyecto ANID-COVID1012 "Desarrollo e implementación de procedimientos docentes para facilitar la disposición al aprendizaje en condiciones de distanciamiento físico por pandemia de Covid-19, en asignaturas de primer año universitario con mediano o alto riesgo de fracaso".

\section{REFERENCIAS}

Britton, B.K., y Tesser, A., Effects of Time-Management Practices on College Grades, https://doi.org/10.1037/00220663.83.3.405, Journal of Educational Psychology, 83(3), 405-410 (1991)

Broc, M., Voluntad para Estudiar, Regulación del Esfuerzo, Gestión Eficaz del Tiempo y Rendimiento Académico en Alumnos Universitarios, Revista de Investigación Educativa, ISSN 1989-9106, 29(1), 171-185 (2011)

Caicedo, E., y Zalazar-Jaime, M., Entrevistas Cognitivas: Revisión, Directrices de Uso y Aplicación en Investigaciones Psicológicas, https://dx.doi.org/10.15689/ap.2018.1703.14883.09, Avaliação Psicológica, 17(3), 362-370 (2018)

Carney, C., McNeish, S., y McColl, J., The Impact of Part Time Employment on Students' Health and Academic Performance: a Scottish Perspective, https://doi.org/10.1080/03098770500353300, Journal of Further and Higher Education, 29(4), 307-319 (2005)

Cortina, J.M., What is coefficient alpha? An Examination of Theory and Applications, https://doi.org/10.1037/00219010.78.1.98, Journal of Applied Psychology, 78(1), 98-104 (1993)

Credé, M., y Kuncel, N.R., Study Habits, Skills, and Attitudes: The Third Pillar Supporting Collegiate Academic Performance, https://doi.org/10.1111/j.1745-6924.2008.00089.x, Perspectives on Psychological Science, 3(6), 425-453 (2008)

Credé, M., y Niehorster, S., Adjustment to College as Measured by the Student Adaptation to College Questionnaire: A Quantitative Review of its Structure and Relationships with Correlates and Consequences, https://doi.org/10.1007/s10648-011-9184-5, Educational Psychology Review, 24, 133-165 (2012)

Creswell, J.W., y Clark, V.L., Designing and Conducting Mixed Methods Research, 3a Ed., ISBN: 9781483344379, 137184, Sage, Los Angeles, USA (2018) 
De la Barrera, M.L., Donolo, D.S., y Rianudo, M.C., Ritmo de Estudio y Trayectoria Universitaria, Anales de Psicología, ISSN 1695-2294, 24(1), 9-15 (2008)

De La Fuente, J., López-García, M., y otros 3 autores, Personal Self-Regulation, Learning Approaches, Resilience and Test Anxiety in Psychology Students, http://dx.doi.org/10.15581/004.32.9-26, Estudios sobre Educación, 32, 9-26 (2017)

Díaz-Mujica, A., Pérez-Villalobos, M.V., y otros 3 autores, Affective and Cognitive Variables Involved in Structural Prediction of University Dropout, https://doi.org/10.7334/psicothema2019.124, Psicothema, 31(4), 429-436 (2019)

Durán-Aponte, E., y Pujol, L., Manejo del Tiempo Académico en Jóvenes que Inician Estudios en la Universidad Simón Bolívar, Revista Latinoamericana de Ciencias Sociales, Niñez y Juventud, ISSN 2027-7679, 11(1), 93-108 (2013)

Elvira-Valdés, M., y Pujol, L., Autorregulación y Rendimiento Académico en la Transición Secundaria-Universidad, Revista Latinoamericana de Ciencias Sociales, Niñez y Juventud, ISSN 2027-7679, 1(10), 709-720 (2012)

Fong, C.J., Davis, C.W., y otros 4 autores, Psychosocial Factors and Community College Student Success: A MetaAnalytic Investigation, https://doi.org/10.3102/0034654316653479, Review of Educational Research, 87(2), 388-424 (2017)

Flores, J.G., Jiménez, E.G., y Gómez, G.R., Análisis de Respuestas Libres en los Cuestionarios. El Método de las Especificidades, Revista Investigación Educativa, ISSN 1989-9106, 14(1), 129-147 (1996)

Garzón-Umerenkova, A., y Gil-Flores, J., Gestión del Tiempo y Procrastinación en la Educación Superior, http://dx.doi.org/10.11144/Javeriana.upsy16-3.gtpe, Universitas Psychologica, 16(3), 124-136 (2017)

Huertas, A., Vesga, G., y Galindo, M., Validación del Instrumento 'Inventario de Habilidades Metacognitivas (MAI)' con Estudiantes Colombianos, Praxis \& Saber, ISSN 2216-0159, 5(10), 56-74 (2014)

López-Angulo, Y., Pérez-Villalobos, M. V., Cobo-Rendón, R. C., y Díaz-Mujica, A.E., Apoyo social, sexo y área del conocimiento en el rendimiento académico autopercibido de estudiantes universitarios chilenos, http://dx.doi.org/10.4067/S0718-50062020000300011, Form Univ, 13(3), 11-18 (2020)

Pérez, M., Díaz-Mujica, A., González-Pienda, J., y Núñez, J., Docencia para Facilitar el Aprendizaje Activo y Autorregulado, http://dx.doi.org/10.7213/rde.v10i30.2509, Revista Diálogo Educacional, 10(30), 409-424 (2010)

Pérez, M.V., Valenzuela, M., y otros 3 autores, Dificultades de Aprendizaje en Estudiantes Universitarios de Primer Año, Atenea, ISSN 0718-0462, 508, 135-150 (2013)

Robbins, S.B., Lauver, K., y otros 4 autores, Do Psychosocial and Study Skill Factors Predict College Outcomes? A Meta-analysis, https://doi.org/10.1037/0033-2909.130.2.261, Psychological Bulletin, 130(2), 261-288 (2004)

Robotham, D., Student Part-Time Employment: Characteristics and Consequences, http://dx.doi.org/10.1108/00400911211198904, Education + Training, 54(1), 65-75 (2012)

Sainz, M., Ferrero, A.M., y Ugidos, A., Time Management: Skills to Learn and Put into Practice, https://doi.org/10.1108/ET-01-2018-0027, Education + Training, 61(5), 635-648 (2019)

Schraw, G., y Dennison, R.S., Assessing Metacognitive Awareness, https://doi.org/10.1006/ceps.1994.1033, Contemporary Educational Psychology, 19(4), 460-475 (1994)

Soares, A., Almeida, L., y Guisandez, A., Ambiente Académico y Adaptación a la Universidad: Un Estudio con Estudiantes de $1^{\circ}$ año de la Universidad Do Minho, Revista lberoamericana de Psicología y Salud, ISSN 1989-9246, 2(1), 99-121 (2011)

Umerenkova, A.G., y Flores, J., Gestión del Tiempo en Alumnado Universitario con Diferentes Niveles de Rendimiento Académico, https://doi.org/10.1590/s1678-4634201708157900, Educação e Pesquisa, 44, 1-16 (2018)

Zimmerman, B.J., Becoming a Self-Regulated Learner: An Overview, https://doi.org/10.1207/s15430421tip4102_2, Theory into Practice, 41(2), 64-70 (2002)

Zimmerman, B.J., y Bandura, A., Impact of Self-Regulatory Influences on Writing Course Attainment, http://dx.doi.org/10.2307/1163397, American Educational Research Journal, 31(4), 845-862 (1994) 